\title{
CUERPO EDITORIAL
}

\section{DIRECTOR}

- Dr. Esteban Sanchez Gaitan, Hospital San Vicente de Paúl, Heredia, Costa Rica.

\section{CONSEJO EDITORIAL}

- Dr. Cesar Vallejos Pasache, Hospital III lquitos, Loreto, Perú

- Dra. Anais López, Hospital Nacional Edgardo Rebagliati Martins, Lima, Perú.

- Dra. Ingrid Ballesteros Ordoñez, Pontificia Universidad Javeriana, Bogotá, Colombia.

- Dra. Mariela Burga, Hospital Nacional Edgardo Rebagliati Martins. Lima, Perú.

- Dra. Patricia Santos Carlín, Ministerio de Salud (MINSA). Lima, Perú.

- Dr. Raydel Pérez Castillo, Centro Provincial de Medicina Deportiva Las Tunas, Cuba.

\section{COMITÉ CIENTÍFICO}

- $\quad$ Dr. Zulema Berrios Fuentes, Ministerio de Salud (MINSA), Lima, Perú.

- $\quad$ Dr. Gerardo Francisco Javier Rivera Silva, Universidad de Monterrey, Nuevo León, México.

- $\quad$ Dr. Gilberto Malpartida Toribio, Hospital de la Solidaridad, Lima, Perú.

- Dra. Marcela Fernández Brenes, Caja costarricense del Seguro Social, Limón, Costa Rica

- $\quad$ Dr. Hans Reyes Garay, Eastern Maine Medical Center, Maine, United States.

- $\quad$ Dr. Steven Acevedo Naranjo, Saint- Luc Hospital, Quebec, Canadá.

- $\quad$ Dr. Luis Osvaldo Farington Reyes, Hospital regional universitario Jose Maria Cabral y Baez, Republica Dominicana.

- Dra.Caridad Maria Tamayo Reus, Hospital Pediátrico Sur Antonio María Béguez César de Santiago de Cuba, Cuba.

- $\quad$ Dr. Luis Malpartida Toribio, Hospital Nacional Daniel Alcides Carrión, Callao, Perú.

- Dra. Allison Viviana Segura Cotrino, Médico Jurídico en Prestadora de Salud, Colombia.

- Mg.Luis Eduardo Traviezo Valles, Universidad Centroccidental "Lisandro Alvarado" (UCLA), Barquisimeto, Venezuela.

- Dr.Pablo Paúl Ulloa Ochoa, Instituto Oncológico Nacional "Dr. Juan Tanca Marengo", Guayaquil, Ecuador.

\section{EQUÍPO TÉCNICO}

- Msc. Meylin Yamile Fernández Reyes, Universidad de Valencia, España.

- $\quad$ Lic. Margarita Ampudia Matos, Hospital de Emergencias Grau, Lima, Perú.

- $\quad$ Ing. Jorge Malpartida Toribio, Telefónica del Perú, Lima, Perú.

- $\quad$ Srta. Maricielo Ampudia Gutiérrez, George Mason University, Virginia, Estados Unidos.

\section{EDITORIAL ESCULAPIO}

50 metros norte de UCIMED, Sabana Sur, San José-Costa Rica Teléfono: 8668002

E-mail: revistamedicasinergia@gmail.com

\section{ENTIDAD EDITORA}

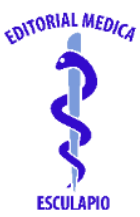
SOMEA SOCIEDAD DE MEDICOS DE AMERICA

Frente de la parada de buses Guácimo, Limón. Costa Rica Teléfono: 8668002 Sociedaddemedicosdeamerica@hotmail.com https://somea.businesscatalyst.com/informacion.html 


\title{
Actualización de cardiomiopatía de Takotsubo
}

\author{
Takotsubo cardiomyopathy actualization
}

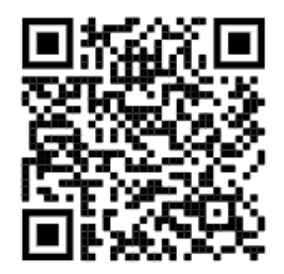

RECIBIDO

$27 / 01 / 2020$

\author{
${ }^{1}$ Dra. Marilyn Campos Quesada \\ Investigadora independiente, San José, Costa Rica \\ (iD) https://orcid.org/0000-0001-6222-3769 \\ ${ }^{2}$ Dr. Daniel Molina Castaño \\ Investigador independiente, San José, Costa Rica \\ (iD) https://orcid.org/0000-0003-0409-5839
}

${ }^{3}$ Dr. Alberto Núñez Guerrero Investigar independiente, San José, Costa Rica

(iD https://orcid.org/0000-0002-3611-3600

\begin{tabular}{|c|c|}
\hline & $\begin{array}{c}\text { CORREGIDO } \\
\text { 10/02/2020 }\end{array}$ \\
\hline $\begin{array}{l}\text { 19édico general, graduada } \\
\text { de la Universidad de } \\
\text { Ciencias } \\
\text { (UCIMED), } \\
\text { cod. MED16060 } \\
\text { marilyncampos94@hotmail.com } \\
\text { } 2 \text { Médicas } \\
\text { Médico general, graduado } \\
\text { de la Universidad de } \\
\text { Ciencias Médicas } \\
\text { (UCIMED), } \\
\text { cod. MED16131. } \\
\text { damo rfl@hotmail.com }\end{array}$ & $\begin{array}{l}\text { RESUMEN } \\
\text { El síndrome de Takotsubo es una cardiomiopatía inducida por estrés físic } \\
\text { o emocional, caracterizado por ser una dilatación apical ventricular, qu } \\
\text { afecta predominantemente a mujeres posmenopáusicas, sus mecanismo } \\
\text { fisiopatológicos precisos aún son desconocidos. Inicialmente fu } \\
\text { considerada un cuadro benigno; sin embargo, actualmente se conoce qu } \\
\text { está asociada a severas complicaciones clínicas, entre ellas la muerte. Est } \\
\text { patología puede ser indistinguible del síndrome coronario agudo por I } \\
\text { similitud en manifestaciones clínicas, hallazgos electrocardiográficos y d } \\
\text { laboratorio. La angiografía coronaria con ventriculografía, es considerado } \\
\text { método diagnóstico de elección. Actualmente no se cuenta con una guía d } \\
\text { manejo, no obstante, la meta de tratamiento, se basa en medidas d } \\
\text { soporte y abordaje de las complicaciones asociadas. } \\
\text { PALABRAS CLAVE: cardiomiopatía de Takotsubo; síndrome coronario } \\
\text { agudo; síndrome; shock cardiogénico; insuficiencia cardiaca. }\end{array}$ \\
\hline $\begin{array}{l}3 \text { Médico general, graduado } \\
\text { de la Universidad de } \\
\text { Ciencias } \quad \text { Médicas } \\
\text { (UCIMED), } \\
\text { cod. MED16141. } \\
\text { anunezg94@gmail.com }\end{array}$ & $\begin{array}{l}\text { Takotsubo syndrome is a cardiomyopathy induced by physical or emotion } \\
\text { stress, characterized by being a ventricular apical dilation, whic } \\
\text { predominantly affects postmenopausal women, currently its precis } \\
\text { pathophysiological mechanisms are still unknown. It was initially considere } \\
\text { a benign condition; however, it is currently known to be associated wi }\end{array}$ \\
\hline
\end{tabular}


severe clinical complications, including death. This pathology can be indistinguishable from acute coronary syndrome due to the similarity in clinical manifestations, electrocardiographic and laboratory findings. Coronary angiography with ventriculography is considered the diagnostic method of choice. Currently there is no management guide, however, the treatment goal is based on support measures and addressing associated complications.

KEYWORDS: Takotsubo cardiomyopathy; acute coronary syndrome; syndrome; cardiogenic shock; heart failure.

\section{INTRODUCCIÓN}

El síndrome de Takotsubo fue inicialmente descrito como una miocardiopatía potencialmente reversible, sin embargo, al presente se ha reconocido la asociación de esta con múltiples complicaciones principalmente en el cuadro agudo, por lo que el reconocimiento, abordaje temprano y manejo óptimo por parte del personal médico es fundamental. Fue descrita por primera vez en el decenio de 1990, en Japón y su nombre se debe a la similitud con una herramienta utilizada para atrapar pulpos. Es una patología que en lo últimos años ha presentado un auge, sin embargo, aún está rodeada de muchas incógnitas, especialmente en lo que respecta a su fisiopatología y factores de riesgo. El objetivo de esta revisión bibliográfica, es difundir el conocimiento general de la enfermedad, con la información más actualizada disponible en la comunidad médica, con el fin de disminuir su infra diagnóstico y brindar un manejo adecuado al paciente.

\section{MÉTODO}

La búsqueda de información para esta revisión, se realizó a través de las revistas del Colegio Americano de Cardiología, Sociedad Europea de Cardiología, The New England Journal of Medicine, buscadores como Google Scholar y también se tomaron en cuenta capítulos de las últimas ediciones de los libros Harrison de Medicina Interna, Braunwald de cardiología. Los artículos que se utilizaron no fueron mayor a 5 años de antigüedad, utilizando los siguientes términos para búsqueda: Miocardiopatías, síndrome de Takotsubo, shock cardiogénico, síndrome coronario agudo, fisiopatología takotsubo.

\section{DEFINICIÓN}

Se define como una miocardiopatía con dilatación apical inducida del ventrículo izquierdo en sístole, la cual predomina en el sexo femenino, principalmente pacientes de edad avanzada las cuales fueron sometidas a periodos intensos de estrés agudo, tanto físico como emocional. Anatómicamente existe una dilatación ventricular global con contracción basal, dando semejanza a un instrumento utilizado por pescadores en Japón para atrapar pulpos, por esta razón el origen de su nombre (1-4). 


\section{EPIDEMIOLOGÍA}

Este síndrome se observa en varias razas, sin embargo, es poco común en hispanos y afroamericanos $(4,5)$. La incidencia es complicada de estimar dado que es usualmente infradiagnosticado $(5,6)$. Una de las principales causas es el desconocimiento médico de la enfermedad, aunque en los últimos años ha aumentado probablemente por un mayor entendimiento de la enfermedad y la utilización de métodos invasivos tempranos como la angiografía coronaria invasiva $(5,6)$.

Dentro de los estudios, se reporta alrededor de un $90 \%$ pacientes femeninas, con una edad promedio de 65-70 años. (4-9) No obstante, se ha descrito este síndrome en ambos géneros $y$ en varios grupos etarios incluyendo niños $(4,5)$.

De todos los pacientes que ingresan al servicio de urgencias por manifestaciones sugestivas de síndrome coronario agudo, un $2 \%$ presentan Síndrome Takotsubo (4,5,7-9) y un $10 \%$ aproximadamente si sólo se consideran mujeres. $(4,5,7)$. Este puede recurrir, con rangos que varían de 0-22\% (5).

\section{FISIOPATOLOGÍA}

La fisiopatología exacta de la enfermedad todavía es desconocida, no obstante, se han propuesto diversos mecanismos que expliquen la presentación de la miocardiopatía de Takotsubo (MCT).

La hipótesis más destacada es la liberación excesiva de catecolaminas $(4,7,10)$, que ocasiona una disfunción microvascular regional en pacientes susceptibles, con sobrecarga de calcio intracelular (10). En varios casos se identifica un desencadenante emocional o físico que precipita la miocardiopatía. (4) Los patrones de abombamiento, así como manifestaciones clínicas de este síndrome, pueden ser producidas por la administración intravenosa de beta bloqueadores y catecolaminas. Durante la fase aguda un análisis de la frecuencia cardiaca ha demostrado un aumento en la actividad simpática con una depresión de la parasimpática $(4,5)$.

"A pesar que la estimulación simpática es primordial en la miocardiopatía de Takotsubo, el mecanismo exacto por el cual el exceso de catecolaminas precipita el aturdimiento miocárdico en los diferentes patrones de abombamiento es desconocido. "(4) Los principales mecanismos que se han propuesto incluyen la ruptura de placa, toxicidad por catecolaminas en cardiomiocitos, disfunción microcirculatoria, espasmo multivaso epicárdico y activación de vías de supervivencia miocárdica $(4,5)$.

$\mathrm{La}$ isquemia transitoria producto de la ruptura de placa puede ocasionar un aturdimiento miocárdico en aquellos pacientes con enfermedad arterial coronaria no obstructiva (4).

$\mathrm{Se}$ han reportado placas ateroescleróticas en la porción media de la arteria descendente anterior izquierda, sin embargo, el ultrasonido intravascular y la tomografía de coherencia óptica no logran identificar ruptura de placas en la mayoría de pacientes con miocardiopatía de Takotsubo.

Esta teoría es poco probable, dado que las anormalidades del movimiento de pared se extienden más allá de un solo territorio arterial coronario, en algunos 
casos incluyendo el ventrículo derecho (4).

La miocardiopatía de Takotsubo puede estar asociada a una disfunción endotelial (4,9). A pesar que la vasoconstricción epicárdica coronaria puede contribuir con esta miocardiopatía en un grupo de pacientes, en la gran mayoría no se observa un espasmo epicárdico a pesar de la utilización de agentes provocantes $(4,5,7,9)$.

"La endotelina y catecolaminas ejercen su efecto vasoconstrictor a nivel de la microvasculatura coronaria, donde hay una predominancia de los receptores a1 y receptores de endotelina tipo A, por lo que se sugiere una disfunción microcirculatoria"(4). Se ha comprobado que tiene un rol fundamental en la MCT dado que en la fase aguda de la enfermedad, la administración de adenosina mejora la perfusión miocárdica transitoriamente, además de mejorar la fracción de eyección ventricular izquierda y el Wall motion score index $(4,9)$. Esta propuesta es respaldada por los hallazgos de las biopsias endomiocárdicas donde se observaron apoptosis de las células endoteliales microvasculares. (4).

La disfunción transitoria del ventrículo izquierdo puede ser el resultado de los efectos directos de las catecolaminas, evidenciado en las biopsias endomiocárdicas, donde eventualmente se han encontrado necrosis de bandas de contracción. $(4,5,7,9)$ Este tipo de necrosis se observa generalmente en pacientes con exposición excesiva a las catecolaminas, como un feocromocitoma o hemorragias subaracnoideas. $(4,7,9)$ Estos pacientes presentan un aumento en la susceptibilidad de los cardiomiocitos y la microcirculación a las hormonas estresoras lo que conlleva a una disfunción del ventrículo izquierdo transitoria pero prolongada, con una inflamación miocárdica secundaria. (4) La anormalidad transitoria severa de la movilidad de pared observada en MCT, es sugestiva de que mecanismos protectores jueguen un papel en preservar la integridad miocárdica. $(4,9)$ Se establecen 2 mecanismos probables de la protección miocárdica, uno siendo relacionado a adrenoreceptores y la otra la vía supervivencia fosfoinositida quinasa 3/proteína quinasa B (AKT) $(4,9)$.

El primer mecanismo va a producir un efecto inotrópico negativo al cambiar la proteína GS a Gi en el B2 adrenoreceptor en respuesta a supraniveles de epinefrina $(4,5,7,9)$ El segundo mecanismo involucra la supervivencia celular y aumentando la biosíntesis de proteínas. $(4,7,9)$ "Estos dos factores son esenciales para la regeneración miocárdica y mejora del funcionamiento ventricular izquierdo"(7).

\section{FACTORES DE RIESGO}

Todos los individuos se enfrentarán a estrés emocional o físico a lo largo de la vida, sin embargo, es un grupo minoritario el que a consecuencia de esto va a desarrollar el síndrome y a un número menor el que tendrá recurrencia. Se ha relacionado la existencia de factores de riesgo causantes de mayor susceptibilidad (11):

- Sexo: es de los factores de riesgo más conocidos, siendo predominante entre mujeres posmenopáusicas, con una incidencia 9:1, no obstante, Japón es la excepción, siendo más frecuente en hombres por razones 
que aún son poco claras. Esta diferencia tan marcada sugiere que existe una influencia hormonal, siendo la disminución de estrógenos un potencial factor de riesgo. Es conocido que lo estrógenos confieren una protección mayor en mujeres perimenopaúsicas, estos atenúan la vasoconstricción mediada por catecolaminas y disminuyen la respuesta simpática al estrés emocional. Mujeres mayores de 55 años tienen 5 veces mayor riesgo comparado con aquellas de menor edad $(4,8,11,12)$.

- Genética: a pesar de que el síndrome no se considera una miocardiopatía genética primaria, se ha sugerido un rol genético posterior a un reporte de 5 casos familiares en el que se involucraban 3 pares de hermanas y 2 casos madre-hija, por lo que se cree que existe la posibilidad de que alelos susceptibles recesivos interactúen con factores ambientales $(4,11)$.

- Padecimientos psiquiátricos y neurológicos: tienen una alta prevalencia entre pacientes con esta miocardiopatía, se estima que $27 \%$ de estos pacientes son portadores de enfermedades neurológicas agudas 0 crónicas y $42 \%$ de ellos padecen trastornos psiquiátricos, siendo más común y estando presente en alrededor de la mitad de los pacientes la depresión $(4,11,13)$.

- Diabetes mellitus: el rol de esta patología en la miocardiopatía por estrés es controversial, algunos estudios afirmar que debido a la remodelación neuroanatómica de la patología y a la activación de ciertos neuropéptidos suele predisponer a la miocardiopatía y que esta se asocia a una mayor tasa de mortalidad.(8) Sin embargo, recientes estudios han postulado que esta, podría ser un factor protector y sugieren que pacientes portadores de diabetes tienen pronósticos más favorables intrahospitalarios y en el primer año posterior al cuadro agudo (6).

- Emociones positivas: el papel que desempeñan estas es menos claro, algunos estudios las han relacionado con menor riesgo cardiovascular, mientras que otros, por el contrario, indican que aumentan el riesgo de padecer síndromes coronarios agudos. Curiosamente se ha demostrado que el día de cumpleaños una persona aumenta el riesgo en $27 \%$ de padecer isquemia miocárdica en comparación de otros días (11).

- Cannabis: recientes investigaciones han dado a conocer una relación entre su uso y el riesgo de desarrollar síndrome de Takotsubo, el consumo exógeno de esta provoca a nivel miocárdico una estimulación similar a estados hipercatecolaminérgico. Este factor de riesgo cada día toma más importancia debido al creciente consumo de esta sustancia, tanto recreacional como por prescripción médica. $(8,14)$

\section{MANIFESTACIONES CLÍNICAS}

En la fase aguda las manifestaciones clínicas son muy similares y prácticamente indistinguibles clínicamente de un síndrome coronario agudo, el síntoma predominante es el 
dolor torácico en más del $75 \%$ de los pacientes, seguido de disnea y síncope $(4,6,8,11,15)$. Entre las manifestaciones menos frecuentes, pero con igual relevancia médica debido a la gravedad de las condiciones, son el edema agudo de pulmón, choque cardiogénico, arritmias ventriculares e incluso paro cardiaco, además de estos se han descrito otros menos específicos como sensación de debilidad, tos y fiebre. En algunas situaciones el paciente puede cursar asintomático y ser diagnosticados de manera incidental cuando presentan cambios nuevos en el electrocardiograma 0 por elevaciones súbitas de biomarcadores cardiacos $(11,15)$. Cuando la causa de la miocardiopatía es inducida por estrés físico severo los síntomas y signos clínicos que suelen predominar son los de la enfermedad de fondo, por ejemplo, en pacientes en los que el factor precipitante son eventos cerebro vasculares o convulsiones, el dolor torácico y las palpitaciones son menos frecuentes y estos suelen presentarse con alteración del estado de consciencia, deterioro neurológico 0 inestabilidad hemodinámica (4).

\section{DIAGNÓSTICO}

El diagnóstico de MCT puede presentar un reto clínico dado la similitud de presentación con un infarto agudo de miocardio (IAM). $(4,5)$ Es imprescindible realizar una historia clínica completa un examen físico cardiovascular y estudios complementarios como electrocardiograma (EKG), ecocardiograma y angiografía coronaria $(8,11)$. La angiografía coronaria con ventriculografía izquierda se considera el tratamiento de referencia (gold standard) para confirmar o excluir una MCT $(4,8)$. Dado que no existe un consenso general en la enfermedad de Takotsubo, recientemente se ha propuesto la InterTAK Diagnostic Criteria, este consta de 8 criterios diagnósticos, los cuales de presentar alguno se asocia a un mayor riesgo de portar la enfermedad. Estos criterios son $(4,8)$ :

1. Los pacientes muestran una disfunción ventricular izquierda transitoria (hipocinesia, acinesia o discinesia) como abombamiento apical ventricular, o anormalidades en movilidad de pared (basal, focal o medioventricular). Puede ocurrir transición entre los patrones anormales de movimiento de pared. Además, puede involucrar el ventrículo derecho. La anormalidad de movimiento de pared se extiende usualmente más allá de una sola distribución vascular epicárdica, sin embargo, pueden ocurrir casos en los cuales se mantenga la anormalidad en una sola región vascular (MCT focal).

2. Un estrés físico, emocional o combinación de ambos, pueden desencadenar un evento del síndrome de Takotsubo, sin embargo, no es una obligación.

3. Feocromocitoma o desórdenes neurológicos (evento cerebrovascular, convulsiones o hemorragias subaracnoideas), pueden desencadenar una miocardiopatía Takotsubo.

4. Estan presentes nuevas anormalidades en el EKG (elevación del segmento ST, depresión del segmento ST, inversión onda $\mathrm{T} O$ 
prolongación QT), aunque pueden existir casos en los cuales no presenta ninguna alteración electrocardiográfica.

5. Niveles de biomarcadores cardiacos como troponina o creatin-kinasa, están moderadamente elevados en la mayoría de casos, es común una elevación significativa del péptido natriurético cerebral.

6. Una enfermedad arterial coronaria significativa no es una contradicción de síndrome Takotsubo.

7. Mujeres posmenopáusicas son afectadas predominantemente.

8. Los pacientes no tienen evidencia de una miocarditis infecciosa.

Aunado a estos criterios diagnósticos, el Registro internacional de Takotsubo crea el InterTAK Diagnostic Score, una herramienta con la capacidad de estimar la probabilidad de padecer la miocardiopatía y a su vez distinguir en fases tempranas de síndromes coronarios agudos, con una alta sensibilidad y especificidad diagnóstica. En la TABLA 1 se menciona los parámetros y puntaje. (16). El puntaje máximo es de 100 puntos. Cada parámetro tiene un puntaje dependiente de su importancia diagnóstica (16). Dependiendo de la prevalencia de la enfermedad, un puntaje de 30 tiene una probabilidad de $<1 \%$, si presentan 50 puntos presentan una probabilidad de $18 \%$, mientras que un puntaje $>70$ tienen una probabilidad de alrededor de $90 \%$ de presentar una MCT $(8,16)$. Los pacientes con un puntaje menor a 70, deberían someterse a una angiografía coronaria con ventriculografía izquierda, mientras que aquellos con un puntaje mayor a 70 , debería considerarse una ecocardiografía transtorácica (16).

\section{TABLA 1. InterTAK Diagnostic Score}

\begin{tabular}{|l|c|}
\hline \multicolumn{1}{|c|}{ Parámetro } & Puntaje \\
\hline Sexo femenino & 25 \\
\hline Estrés emocional & 24 \\
\hline Estrés físico & 13 \\
\hline $\begin{array}{l}\text { Ausencia de depresión del } \\
\text { segmento ST (excepto aVR) }\end{array}$ & 12 \\
\hline Alteraciones psiquiátricas & 11 \\
\hline $\begin{array}{l}\text { Desórdenes neurológicos } \\
\text { (hemorragia subaracnoidea, } \\
\text { accidente cerebrovascular, } \\
\text { convulsiones) }\end{array}$ & 9 \\
\hline $\begin{array}{l}\text { Prolongación del intervalo QT } \\
\text { Iro puntos } \\
\text { Probabilidad baja/intermedia de } \\
\text { MCT }\end{array}$ & $\begin{array}{l}\text { > 70 puntos } \\
\text { Probabilidad } \\
\text { alta de MCT }\end{array}$ \\
\hline $\begin{array}{l}\text { FUENTE: Ghadri J-R, Wittstein IS, Prasad A, Sharkey S, Dote K, } \\
\text { Akashi YA, et al. International Expert Consensus Document on } \\
\text { Takotsubo Syndrome (Part II): Diagnostic workup, Outcome and } \\
\text { Management. }\end{array}$ \\
\hline
\end{tabular}

\section{TRATAMIENTO}

Actualmente no se cuenta con una guía sobre el manejo de MCT dado la ausencia de estudios clínicos prospectivos en esta población $(5,8,11,16)$. Por lo tanto, las estrategias terapéuticas son basadas en experiencia clínica y opinión de expertos (16). En el ámbito prehospitalario, estos pacientes deberían de manejarse según las guías de síndrome coronario agudo(SCA), y recibir el tratamiento correspondiente (aspirina, heparina, oxígeno) dado la dificultad de diferenciación clínica de ambas entidades (16).

La meta del tratamiento en estos 
pacientes se basa en cuidados de apoyo y tratamiento de complicaciones respectivas. $(5,8)$ En casos leves, además del tratamiento de soporte, se puede considerar beta bloqueadores y aspirina (5). Como consideración general, estos pacientes deben ser admitidos a una unidad cardiaca o médica para monitorización continua de EKG dado el riesgo de arritmias (particularmente en contexto de QT prolongado) (8). En ausencia de prolongación de QT, la monitorización por 48 horas es suficiente (8).

En aquellos pacientes con una complicación por falla cardiaca, usualmente se inicia el tratamiento con inhibidores de la enzima convertidora de angiotensina(IECAs)/los antagonistas de los receptores de la angiotensina II (ARAII), beta bloqueadores y diuréticos. $(5,8,16)$. Los beta bloqueadores deben utilizarse con cuidado, especialmente en bradicardia y en aquellos con intervalo QT corregido (QTc) >500 m (16).

La nitroglicerina puede mejorar las presiones de llenado del ventrículo derecho e izquierdo, y poscarga en el contexto de una falla cardiaca aguda, sin embargo, puede empeorar en los casos con una obstrucción del tracto de salida del ventrículo izquierdo por lo que se debe evitar (16).

Los medicamentos que prolongan el QT, se deben utilizar con cautela en la fase aguda, dado el riesgo de desarrollar Torsades de Pointes, fibrilación ventricular o taquicardia ventricular (16).

Una disfunción ventricular izquierda severa con abombamiento apical extensa puede predisponer al desarrollo de trombo y subsecuentemente un embolismo sistémico $(5,8,16)$. A pesar de la ausencia de evidencia, el tratamiento con heparina podría ser apropiado, además, el uso de un tratamiento anticoagulante 0 antiplaquetario al egreso puede considerarse por 2-3 meses, siempre individualizando cada caso $(5,8,16)$.

Actualmente no hay evidencia para guiar en cuanto al tratamiento a largo plazo y prevención de recurrencias $(5,8)$. En un análisis retrospectivo, el uso de IECAs/ARA-II se asoció a un beneficio de 1 año en supervivencia $(5,8,16)$. Los bloqueadores de canales de calcio se han asociado con una recuperación rápida y corta (8.) Dado la prevalencia de desórdenes psiquiátricos (depresión, ansiedad), estos pacientes deben tener una valoración inicial (y posterior referencia) para el tratamiento de sus patologías subyacentes $(8,16)$.

\section{COMPLICACIONES}

La función del ventrículo izquierdo en la gran mayoría de los casos regresa a su función normal al cabo de unas semanas, sin embargo, existen complicaciones que pueden ocurrir durante este proceso $(8,11)$.

La insuficiencia cardiaca sistólica es la complicación más frecuente, afecta aproximadamente al $12-45 \%$ de los pacientes. Los predictores de riesgo más comunes son: la edad avanzada, fracción de eyección izquierda baja, niveles de troponinas altos, desencadenante físico vs emocional, entre otros $(8,16)$.

Dentro de las complicaciones más comunes se encuentran la obstrucción del tracto de salida del ventrículo izquierdo (10-25\%), insuficiencia mitral (14-25\%) y el shock cardiogénico (6$20 \%$ ). Otras menos f frecuentes son la fibrilación atrial, trombos ventriculares, 
arresto cardiaco y distintas arritmias, estas son parte de las complicaciones más temidas, por lo que es importante predecir la aparición intra hospitalaria para que se ofrezca un abordaje temprano, cuidadoso y la correcta estratificación de riesgo de los pacientes quienes tengan condición de egreso. $(8,11,16)$.

\section{PRONÓSTICO}

Pese a ser conocida como una patología de condición benigna y reversible, se ha reportado una tasa de mortalidad de aproximadamente $5 \%$ intrahospitalaria. En la mayoría de los casos los pacientes presentaban un cuadro inicial de inestabilidad hemodinámica, asociado principalmente a arresto cardiaco o shock cardiogénico $(8,16)$.

En la actualidad se conoce que la miocardiopatía de Takotsubo es una condición médica que representa una amenaza a la vida del paciente que la porta, incluso se ha demostrado que las complicaciones intra hospitalarias son comparables con las de pacientes diagnosticados con síndrome coronario agudo $(6,8,16)$.

A pesar de no estar del todo claro, datos recientes sugieren que existe una asociación entre el factor desencadenante y el pronóstico del paciente. Se ha propuesto que en quienes no se logra identificar una causa o cuando el desencadenante es estrés emocional tienen mejor pronostico que aquellos pacientes en los que se establece una causa de origen físico, entre estos los más frecuentes son: patología neurológica, feocromocitoma, intervenciones invasivas como cirugía mayor, etc. Sin embargo, estas hipótesis no han sido ampliamente estudiadas (17)

La tasa de recurrencia puede variar, pero se estima que se aproxima a $2-4 \%$ anual, siendo más frecuente que recurra el dolor torácico, pero sin anomalías segmentarias nuevas $(6,8)$.

\section{CONCLUSIONES}

La miocardiopatía de Takotsubo es una patología cardiaca aguda que amerita manejo interdisciplinario debido a sus potenciales complicaciones.

A pesar de ser descrita por primera vez hace 30 años y reconocida como una miocardiopatía hace aproximadamente una década aún sus mecanismos fisiopatológicos y factores de riesgo como la predisposición genética y el rol de la diabetes mellitus no han sido del todo establecidos.

Es una afección extraordinaria por su similitud clínica, hallazgos de laboratorio y electrocardiográficos con el síndrome coronario agudo, principalmente en su fase aguda, por lo tanto, se considera de suma importancia para el personal médico conocerla para evitar sus complicaciones e infra diagnóstico. Se recomienda que ante la sospecha de este cuadro se realicen estudios complementarios como electrocardiograma, ecocardiograma y la angiografía coronaria con ventriculografía izquierda para llevar a cabo un diagnóstico temprano y evitar complicaciones, de esta forma mejorando el pronóstico de los pacientes a nivel intra y extrahospitalario. 


\section{REFERENCIAS}

1. Monstes erín Matesanz C, Piñeiro Otero P, Gonzalez Benito E, López Martínez M, González Cantero JL. Sindrome de Takotsubo. A propósito de un caso. Rev Arg Anestesiol [internet]. 2017 [citado 22 de enero 2020]; 75 (2): 70-74. Disponible en: https://www.elsevier.es/es-revista-revista-argentina-anestesiologia-268articulo-sindrome-takotsubo-a-proposito-un-S0370779217300340

2. Lakdawala NK, Stevenson LW, Loscalzo J. Miocardiopatía y miocarditis. En: Jameson JL, Kasper DL, Longo DL, et al, editores. Harrison principios de medicina interna. Vol2. 20va ed. México: McGraw-Hill; 2018. p. 1779-1796.

3. Sharkey SW. What Medicare Knows About the Takotsubo Cardiomyopathy. JAAC [internet]. 2016 [citado 22 enero 2020]; 4(3): 206-207. Disponible en: http://heartfailure.onlinejacc.org/content/4/3/206

4. Ghadri J-R, Wittstein IS, Prasad A, Sharkey S, Dote K, Akashi YA, et al. International Expert Consensus Document on Takotsubo Syndrome (Part I): clinical characteristics, Diagnostic Criteria, and pathophysiology. ESC [internet]. 2018 [citado el 22 enero 2020]; 39: 2032-2046. Disponible en: https://academic.oup.com/eurhearti/article/39/22/2032/5025412

5. Y-Hassan S, Tornvall P. Epidemiology, pathogenesis, and management of takotsubo syndrome. Clin Auton Res [Internet]. 2018 [citado 22 Ene 2020]; 28(1): 53-65. https://www.ncbi.nlm.nih.gov/pmc/articles/PMC5805795/

6. Núñez-Gil I.J, Mejía-Rentería H.D, Martínez-Losas P. Actualización práctica en síndrome de Takotsubo. Med Clin [Internet]. 2016 [citado el 22 Ene 2020]; 146 (5): e25-e30. Disponible en: https://www.elsevier.es/es-revista-medicina-clinica-2-articulo-actualizacion-practica-sindrome-takotsubo$\underline{\text { S0025775315003218 }}$

7. Akashi Y.J, Nef H.M, Lyon A.R. Epidemiology and pathophysiology of Takotsubo. Nat. Rev. Cardiol [Internet]. 2015 [citado 22 Ene 2020]; 12: 387-397. Disponible en: https://www.nature.com/articles/nrcardio.2015.39

8. De Chazal H.M, Del Buono M.G, Keyser-Marcus L, Ma L, Moeller F.G, Berrocal D et al. Stress Cardiomyopathy Diagnosis and Treatment. JACC [Internet]. 2018 [citado 26 Ene 2018]; 72: 1955-1971. Disponible en: http://www.onlinejacc.org/content/72/16/1955.abstract

9. Pelliccia F, Kaski J.C, Crea F, Camici P.G. Pathophysiology of Takotsubo Syndrome. Circulation [Internet]. 2017 [citado 27 Ene 2020]; 135: 2426-2441. Disponible en: https://www.ahajournals.org/doi/full/10.1161/CIRCULATIONAHA.116.027121

10. Falk R.H, Hershberger R.E. Miocardiopatías dilatada, restrictiva e infiltrante. En: Mann D.L, Zipes D.P, Libby P, Bonow R.O, Tomaselli G.F, Braunwald E, editores. Braunwald tratado de cardiología. Vol 2. 11a ed. España: Elsevier; 2019. p.1580-1601.

11. Morales-Hernández AE, Valencia-López R, Hernández-Salcedo DR, Domínguez-Estrada J. Síndrome de Takotsubo. Med Int Méx [internet] 2016[citado 22 enero 2020]; 32(4): 475-491. Disponible en: https://www.medigraphic.com/pdfs/medintmex/mim-2016/mim164m.pdf

12. Kato K, Lyon AR, Ghadri JR, Templin C. Takotsubo Syndrome: aetiology, presentation and treatment. Heartjnl [internet] 2017 [citado 26 enero 2020]; 103: 1461-1469. Disponible en: https://www.ncbi.nlm.nih.gov/pubmed/28839096

13. Templin C, Ghadri JR, Diekmann J, Napp LC, Bataiosu DR, Jaguszewski M, et al. Clinical Features and outcomes of Takotsubo (stress) Cardiomyopathy. N ENGL J MED [internet] 2015[citado 26 enero 2020];373:929-938. Disponible en:https://ezproxy.ucimed.com:2119/doi/full/10.1056/NEJMoa1406761 
14. Sánchez AM, Sennhauser S, Lozier M, Purow J. Marijuana use and risk of takotsubo cardiomyopathy. JACC

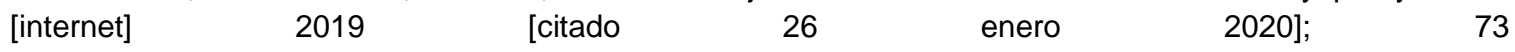
(9):2711.http://www.onlinejacc.org/content/73/9 Supplement 1/2711

15. Arandia-Guzmán J, Antezalana-Llaveta G, Zárate-Carillo A. Miocardiopatía inducida por estrés o síndrome de Takotsubo a proposito de un caso. Gac Med Bol [intenet] 2018; [citado 26 enero 2020]; 21(1): 71-74. Disponible en: $\quad$ http://www.scielo.org.bo/scielo.php?script=sci abstract\&pid=S101229662018000100015\&lng=es\&nrm=iso

16. Ghadri J-R, Wittstein IS, Prasad A, Sharkey S, Dote K, Akashi YA, et al. International Expert Consensus Document on Takotsubo Syndrome (Part II): Diagnostic workup, Outcome and Management. ESC [Internet]. 2018 [citado 26 Ene 2020]; 39: 2047-2062. Disponible en: https://academic.oup.com/eurheart//article/39/22/2047/5025411

17. Ghadri J.R, Kato K, Cammann V.L, Gili S, Jurisic S, Di Vece D et al. Long-Term prognosis of patientes with Takotsubo Syndrome. JAAC [Internet]. 2018 [citado el 23 enero 2020]; 72 (8): 874-882. Disponible en: http://www.onlinejacc.org/content/72/8/874? 